\title{
Numerical Investigation of Tunable Band-passlband-stop Plasmonic Filters with Hollow-core Circular Ring Resonator
}

\author{
Amir Setayesh*, Sayyed Reza Mimaziry, and Mohammad Sadegh Abrishamian \\ Department of Electrical Engineering, K. N. Toosi University of Technology, \\ P.O. Box 16315-1355, Tehran, Iran
}

(Received December 10, 2010 : revised January 28, 2011 : accepted January 28, 2011)

\begin{abstract}
In this paper, we numerically study both band-pass and band-stop plasmonic filters based on MetalInsulator-Metal (MIM) waveguides and circular ring resonators. The band-pass filter consists of two MIM waveguides coupled to each other by a circular ring resonator. The band-stop filter is made up of an MIM waveguide coupled laterally to a circular ring resonator. The propagating modes of Surface Plasmon Polaritons (SPPs) are studied in these structures. By substituting a portion of the ring core with air, while the outer dimensions of the ring resonator are kept constant, we illustrate the possibility of red-shift in resonant wavelengths in order to tune the resonance modes of the proposed filters. This feature is useful for integrated circuits in which we have limitations on the outer dimensions of the filter structure and it is not possible to enlarge the dimension of the ring resonator to reach to longer resonant wavelengths. The results are obtained by a 2D finite-difference time-domain (FDTD) method. The introduced structures have potential applications in plasmonic integrated circuits and can be simply fabricated.
\end{abstract}

Keywords : Plasmonics, MIM waveguide, Band-pass filter, Band-stop filter, Circular ring resonator OCIS codes : (240.6680) Surface Plasmons; (140.4780) Optical resonators; (130.7408) Wavelength filtering devices; (250.5403) Plasmonics; (250.5300) Photonic integrated circuits

\section{INTRODUCTION}

During recent years, plasmonics has been presented as a future technology in integrated circuits incorporating the compactness of electronics and wide bandwidth created by current optical networks [1]. In the region of integrated optics, plasmonic waveguides act as components to guide optical signals to different parts of the circuits. The capability of confining light beyond the diffraction limit and the ability to fabricate devices with dimensions below $100 \mathrm{~nm}$ has promised an evolution in optoelectronic circuits [2]. To guide plasmonic waves for various applications, different geometries have been proposed such as dielectric-loaded surface plasmon polariton waveguides [3], nanostructured plasmonic substrates for enhanced optical biosensing [4], tunable coupled-ring reflector laser diodes [5], dielectric gratings on flat metal surfaces [6, 7], Metal-Insulator-Metal waveguides [8, 9] nano-wires $[10,11]$, chains of nano-particles [12-14], grooves and wedges $[15,16]$, etc. Although some of these nano-guides are inconvenient to implement in optical circuits, MIM waveguides have attracted considerable attention due to simplicity in fabrication and strong field confinement [17].

During recent years, some simple structures have been suggested for plasmonic filters including channel drop filters with disk resonators [18], rectangular geometry resonators $[19,20]$, and ring resonators $[18,21]$. In comparison with complex structures of Bragg reflectors, the aforementioned structures can be fabricated much more easily. Two typical types of plasmonic filters in MIM waveguides are band-pass and band-stop filters. In band-stop filters, majority of the input light spectrum is allowed to pass through the filter and only several wavelengths are prohibited from propagation in the structure. But, the band-pass filters let just some specific wavelengths pass through them and reject the majority of the input light spectrum.

One of the most interesting features of filters is to have tunable resonance wavelengths. In previous works, by varying the outer dimensions of the structure, i.e. cavity length or radius, tunable filters were achieved [21-23]. But in this paper, we want to tune the resonant wavelengths of circular

\footnotetext{
*Corresponding author: setayesh_a@ee.kntu.ac.ir

Color versions of one or more of the figures in this paper are available online.
} 
ring resonator plasmonic filters, without changing the outer size of the structure. Here, by replacing a portion of the ring core with air, we show that tuning characteristics can be attained. As we know, according to some significant limitations on circuit components' dimensions, it may not be possible to enlarge the size of the ring resonator to reach to longer resonant wavelengths. Therefore the new method is beneficial for miniaturizing the filters in the case of integrated circuits. The transmittance characteristics of the proposed filter are presented by 2D FDTD method.

This paper is organized as follows. In Section 2, the fundamental propagation mode of the MIM waveguide is reviewed. In Section 3, firstly a simple band-pass waveguide filter with a circular ring resonator is examined and then, to reach a tunable band-pass filter, a hollow-core ring resonator is introduced. The impact of hollowing out the core of the ring resonator is studied for a band-stop plasmonic filter in Section 4. Finally, Section 5 concludes the paper.

\section{DISPERSION RELATION AND EFFECTIVE REFRACTIVE INDEX OF MIM WAVEGUIDE}

Consider an MIM waveguide structure shown in figure 1. Each of the metal-dielectric interfaces of the waveguide supports a Transverse Magnetic (TM) SPPs mode which propagates along the $x$ direction. It would be anticipated that when the space between the two interfaces is comparable to the decay lengths of SPPs in the dielectric, the SPPs modes become coupled to each other [24]. The field components inside the dielectric layer can be calculated using Maxwell relations

$$
\begin{aligned}
& E_{x}=-i A \frac{1}{\omega \varepsilon_{0} \varepsilon_{1}} k_{1} e^{i \beta x} e^{k_{1} z}+i B \frac{1}{\omega \varepsilon_{0} \varepsilon_{1}} k_{1} e^{i \beta x} e^{-k_{1} z} \\
& E_{z}=A \frac{\beta}{\omega \varepsilon_{0} \varepsilon_{1}} e^{i \beta x} e^{k_{1} z}+B \frac{\beta}{\omega \varepsilon_{0} \varepsilon_{1}} e^{i \beta x} e^{-k_{1} z} \\
& H_{y}=A e^{i \beta x} e^{k_{1} z}+B e^{i \beta x} e^{-k_{1} z}
\end{aligned}
$$

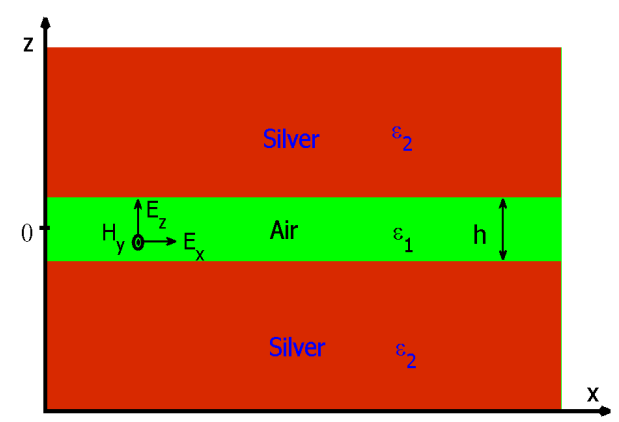

FIG. 1. Schematic of an MIM structure with two semi-infinite metal slabs of permittivity $\varepsilon_{2}$ surrounding a dielectric layer of thickness $h$ and permittivity $\varepsilon_{1}$. where $\beta$, which has a complex value, is the propagation constant of a MIM waveguide and $k_{1}=\sqrt{\beta^{2}-k_{0}^{2}}$ is the wave-vector perpendicular to the propagation direction ( $k_{0}$ is the propagation constant of free-space). For $h<<\lambda$, among different modes which can be supported by MIM, the first odd mode is the only mode that can propagate $(\operatorname{Ex}(z)$ is odd function, $H y(z)$ and $E_{z}(z)$ are even, see figure 1). The corresponding propagation constant of MIM waveguide $\beta$, can be attained from the following dispersion relation $[25,26]$

$$
\tanh \left(\frac{k_{1} h}{2}\right)=-\left(\frac{k_{2} \varepsilon_{1}}{k_{1} \varepsilon_{2}}\right)
$$

Indices 1 and 2 are pointing to dielectric layer and metal slabs, respectively. In order to characterize the dielectric constant of the metal (silver in this study) the seven-pole Drude-Lorentz model is employed. The fitting model is described as $[27,28]$

$$
\varepsilon_{1}(\omega)=1-\frac{\omega_{p}^{2}}{\omega\left(\omega-i \Gamma_{0}\right)}+\sum_{n=1}^{5} \frac{f_{n} \omega_{n}^{2}}{\omega_{n}^{2}-\omega^{2}+i \omega \Gamma_{\mathrm{n}}}
$$

where $\Gamma_{0}=11.5907 \mathrm{THz}$ is the damping constant and $\omega_{\mathrm{p}}$ $=2002.6 \mathrm{THz}$ is the plasma frequency. The quantities of resonant frequencies $\omega_{\mathrm{n}}$, damping constants $\Gamma_{\mathrm{n}}$ and oscillator strength $f_{n}$ are given in Table $1[27,28]$.

TABLE 1. Values of the Drude-Lorentz model parameters (metal is assumed to be silver)[27, 28]

\begin{tabular}{cccc}
\hline \hline$n$ & $\omega_{n}(\mathrm{ev})$ & $\Gamma_{n}(\mathrm{ev})$ & $f_{n}$ \\
\hline 1 & 0.816 & 3.886 & 0.065 \\
2 & 4.481 & 0.452 & 0.124 \\
3 & 8.185 & 0.065 & 0.011 \\
4 & 9.083 & 0.916 & 0.840 \\
5 & 20.29 & 2.419 & 5.646 \\
\hline
\end{tabular}
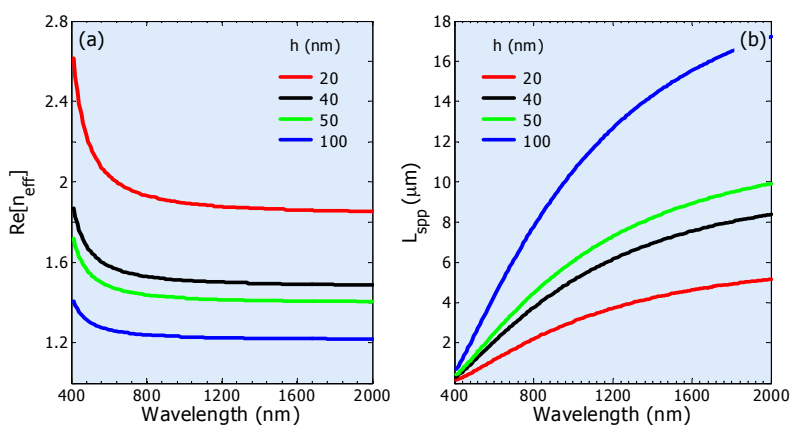

FIG. 2. (a) Real part of effective refraction index as a function of wavelength for four different widths of the air layer in the Ag-air-Ag waveguide. (b) The corresponding propagation length of SPPs as a function of wavelength for the same widths. 
We have analytically calculated the effective refraction index $\left(n_{\text {eff }}=\beta / k_{0}\right)$ for different widths of MIM waveguides, using an IMSL Fortran subroutine by solving Eq. (2). Figure 2 shows the calculated $\operatorname{Re}\left[\mathrm{n}_{\mathrm{eff}}\right]$ and LSPP (propagation length of SPPs defined as $\left.L_{S P P}=1 /(2 \operatorname{Im}[\beta])\right)$, as a function of wavelength.

\section{BAND-PASS PLASMONIC FILTER WITH CIRCULAR RING RESONATOR}

\subsection{Simple Band-pass Waveguide Filter with Circular Ring Resonator}

Figure 3 shows the structure of a simple band-pass plasmonic filter which consists of two MIM waveguides coupled to each other by a circular ring resonator, which was first described in Ref [21]. The filter parameters $h, \Delta, R_{\text {core, }}$ $R_{A v}$, and $R_{\text {out }}$ are the width of MIM waveguide, the gap distance between input/output MIM waveguides and circular ring resonator, the radius of core, the average radius of the ring and the outer radius of the ring resonator, respectively. We set $h=50 \mathrm{~nm}$ which is much smaller than the operating spectrum.

Two power monitors are set at points $\mathrm{P}_{1}$ and $\mathrm{P}_{2}$ to detect the input power $A_{1}$ (without the circular ring resonator) and the transmitted power $\mathrm{A}_{2}$ (with the circular ring resonator). So, the power transmittance is $T=A_{2} / A_{1}[21,27]$. The transmittance of the filter is shownin figure 4(a), assuming $R_{A v}=125 \mathrm{~nm}$ and $\Delta=10 \mathrm{~nm}$. One can see in the figure that there are two resonance peaks at $\lambda \approx 1145 \mathrm{~nm}$ and $\lambda \approx$ $583.5 \mathrm{~nm}$. Figures 4(b), 4(c) depict the field patterns of $\left|\mathrm{H}_{\mathrm{y}}\right|$ for the first and second resonances, respectively. Here, we should note that the resonance wavelengths of the transmission spectrum cannot be achieved simply by $\lambda=\mathrm{L}\left[\operatorname{Re}\left(\mathrm{n}_{\mathrm{eff}}\right) / \mathrm{N}\right]$, where $\mathrm{N}$ is the mode number and $\mathrm{L}=2 \pi \mathrm{R}_{\mathrm{Av}}$ is the length of the ring [21]. The resonant wavelengths of a circular ring resonator are obtained by $[21,29]$

$$
\frac{J_{n}^{\prime}\left(k R_{\text {out }}\right)}{J_{n}^{\prime}\left(k R_{\text {core }}\right)}-\frac{N_{n}^{\prime}\left(k R_{\text {out }}\right)}{N_{n}^{\prime}\left(k R_{\text {core }}\right)}=0
$$

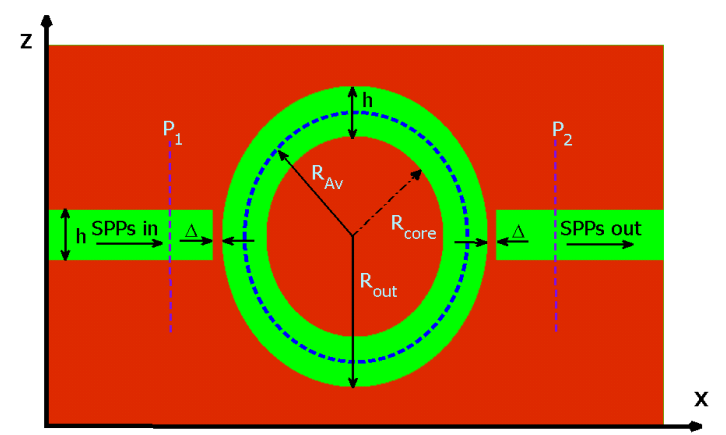

FIG. 3. Schematic of a simple band-pass plasmonic filter consisting of two MIM waveguides coupled to each other by a circular ring resonator. $h$ is set to $50 \mathrm{~nm}$. where $k=\omega\left(\varepsilon_{0} \varepsilon_{\mathrm{r}} \mu_{0}\right)^{1 / 2}$ and $\varepsilon_{\mathrm{r}}=\left(\mathrm{n}_{\mathrm{eff}}\right)^{2} / \mu_{0}$ is the relative permittivity. $\mathrm{J}_{\mathrm{n}}$ is a first kind Bessel function of order $\mathrm{n}$, and $\mathrm{N}_{\mathrm{n}}$ is a second kind Bessel function of order $\mathrm{n}$. So $\mathrm{J}_{\mathrm{n}}^{\prime}$ and $\mathrm{N}_{\mathrm{n}}^{\prime}$ are derivations of the Bessel functions with respect to the argument $(k R)$. Eq. (4) must be numerically solved to determine the resonant wavelengths of the structure. A complete discussion of results obtained by this equation can be found in Ref [21].
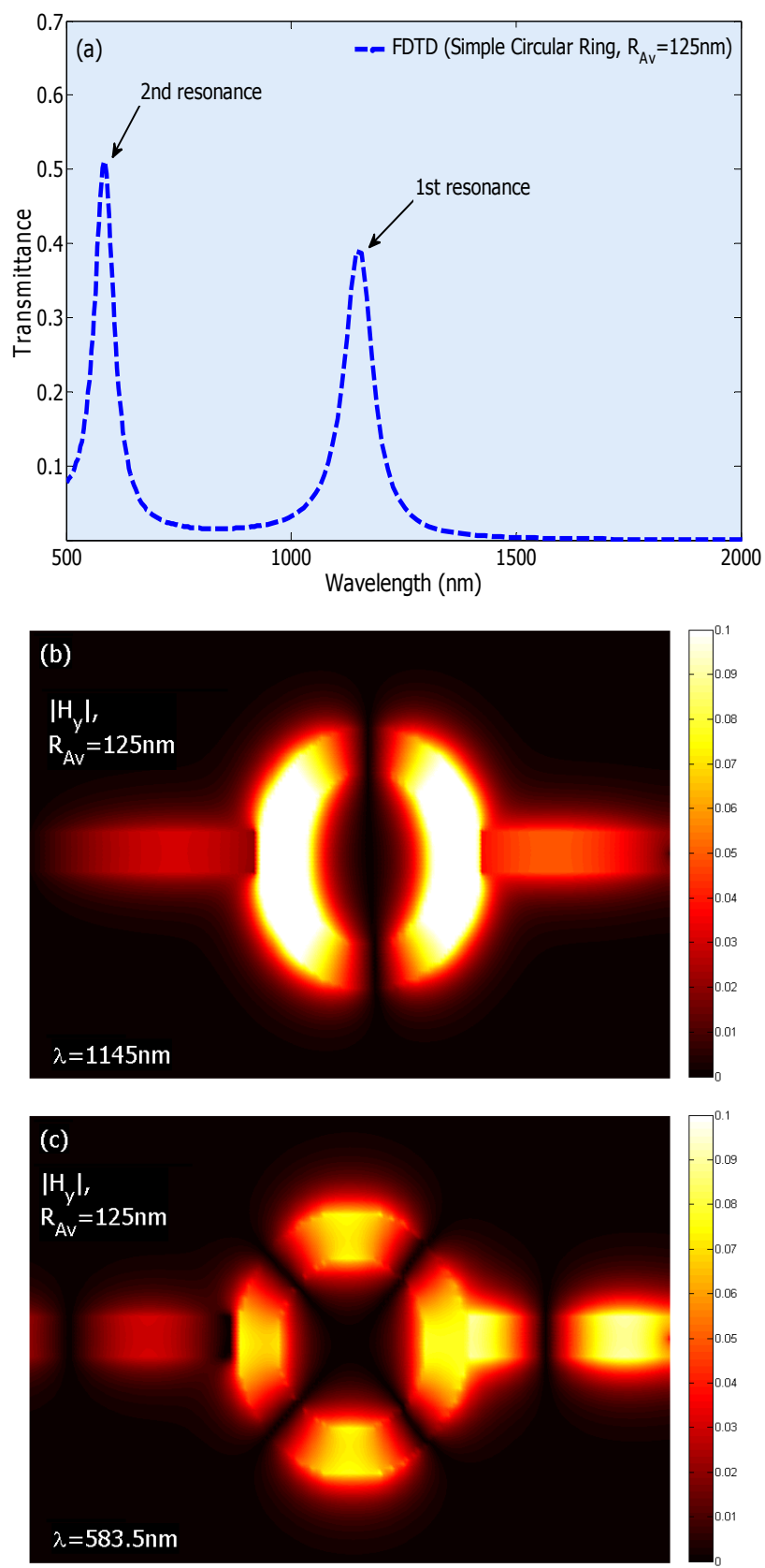

FIG. 4. (a) Transmittance spectrum of the simple band-pass plasmonic filter with circular ring resonator $\left(R_{A v}=125 \mathrm{~nm}, \Delta=\right.$ $10 \mathrm{~nm}$ ). (b) The $\left|\mathrm{H}_{\mathrm{y}}\right|$ field profile of simple band-pass filter at the first resonance wavelength of $\lambda=1145 \mathrm{~nm}$. (c) The $\left|\mathrm{H}_{y}\right|$ field pattern of simple band-pass filter at the second resonance wavelength of $\lambda=583.5 \mathrm{~nm}$. 


\subsection{Band-pass Plasmonic Filter with Hollow-core Ring Resonator}

In this section, we investigate the effect of hollowing out the core of the ring resonator on resonance wavelengths of the band-pass filter while keeping its outer dimensions constant (i.e. $\mathrm{R}_{\mathrm{Av}}, \mathrm{h}$ and $\Delta$ are kept constant). The proposed structure is depicted in figure 5. From the figure one can see that the core of the ring resonator is emptied with the radius of $\mathrm{R}_{\mathrm{H}}$.

Now, we look into the effect of varying $\mathrm{R}_{\mathrm{H}}$ on shifting the

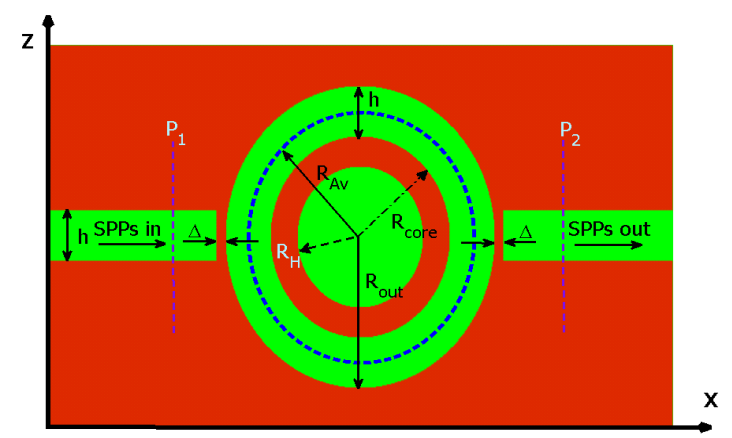

FIG. 5. Schematic of a band-pass plasmonic filter with circular hollow-core ring resonator. $\left(h=50 \mathrm{~nm}, R_{A v}=125 \mathrm{~nm}, \Delta\right.$ $=10 \mathrm{~nm}) . R_{H}$ is variable.
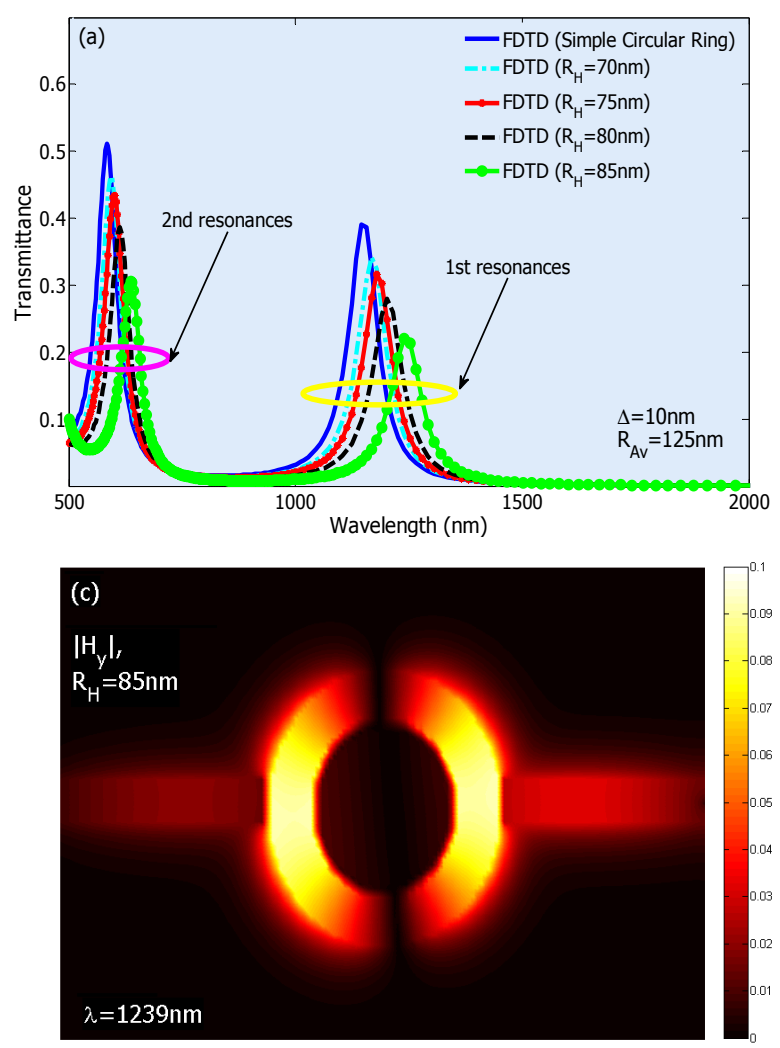

resonant wavelengths of the band-pass filter. The transmittance spectra of the proposed structure is calculated by 2D-FDTD and illustrated in figure 6(a). One can see that by increasing $\mathrm{R}_{\mathrm{H}}$ from $70 \mathrm{~nm}$ to $85 \mathrm{~nm}$, the entire transmission spectrum experiences a red-shift. Figure 6(b) shows that the wavelengthshifts of modes 1 and 2 behave approximately linearly with respect to variation of $\mathrm{R}_{\mathrm{H}}$. This result is in complete accordance with the physical fact that by increasing the resonance volume, resonant wavelength will be increased. According to the transmission curves illustrated in figure 6(a), it is clear that the band-pass filter can be easily tuned by adjusting $R_{H}$.

\subsection{Enhancing the Transmittance Peak}

It is seen from figure 6(a) that by increasing $R_{H}$, the maximum value of the transmittance peak declines. To enhance the transmittance, we decrease the width of ring MIM waveguide in the coupling region. The proposed structure is depicted in figure 7. The procedure of transmittance increment versus $\mathrm{W}$ is illustrated in figure 8(a) for first and second resonance modes of the structure. It is obvious from the figure that the first resonance wavelength has experienced no shifting by varying $\mathrm{W}$, but the second mode is slightly blue-shifted $(\Delta \lambda \approx 7 \mathrm{~nm}$ ) with respect to decreasing $\mathrm{W}$. The relation between decreasing the $\mathrm{W}$ and increment of transmittance for first and second modes is shown in figure 8(b). As can be
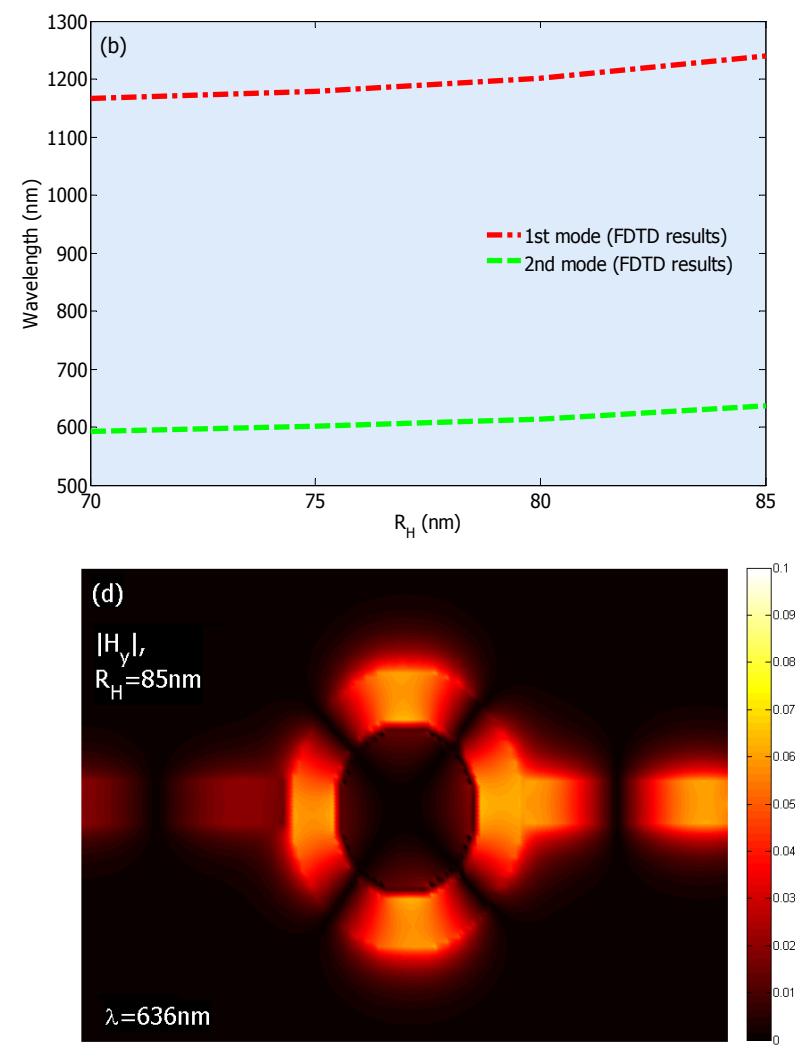

FIG. 6. (a) Transmission spectrum of the band-pass plasmonic filter with circular hollow-core ring resonator shown in figure 5 for different values of $\mathrm{R}_{\mathrm{H}}$. (b) Relationship between resonance wavelengths and hollow radius. (c) The $\left|\mathrm{H}_{\mathrm{y}}\right|$ field profile of band-pass plasmonic filter with circular hollow- core ring resonator at the first resonance wavelength of $\lambda=1239 \mathrm{~nm}$, for $R_{H}=85 \mathrm{~nm}$. (d) The $\left|\mathrm{H}_{\mathrm{y}}\right|$ field pattern of the filter at the second resonance wavelength of $\lambda=636 \mathrm{~nm}$, for $R_{H}=85 \mathrm{~nm}$. 


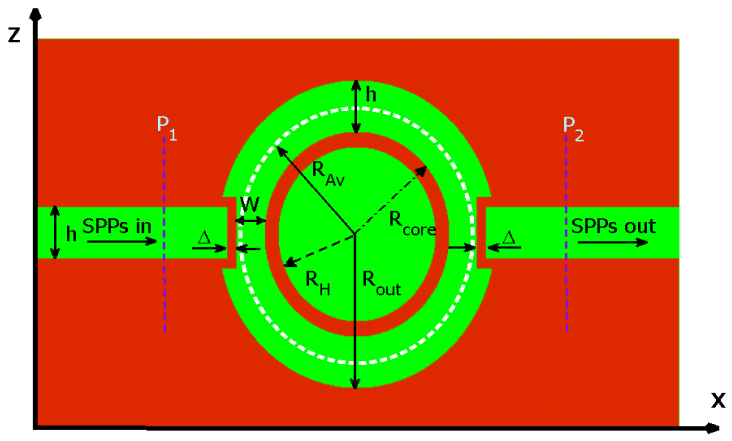

FIG. 7. Schematic of a band-pass plasmonic filter with circular hollow-core ring resonator and reduced width of ring MIM waveguide at the coupling region. ( $h=50 \mathrm{~nm}, R_{A v}=125$ $\left.\mathrm{nm}, R_{H}=85 \mathrm{~nm}, \Delta=10 \mathrm{~nm}\right) . W$ is variable.
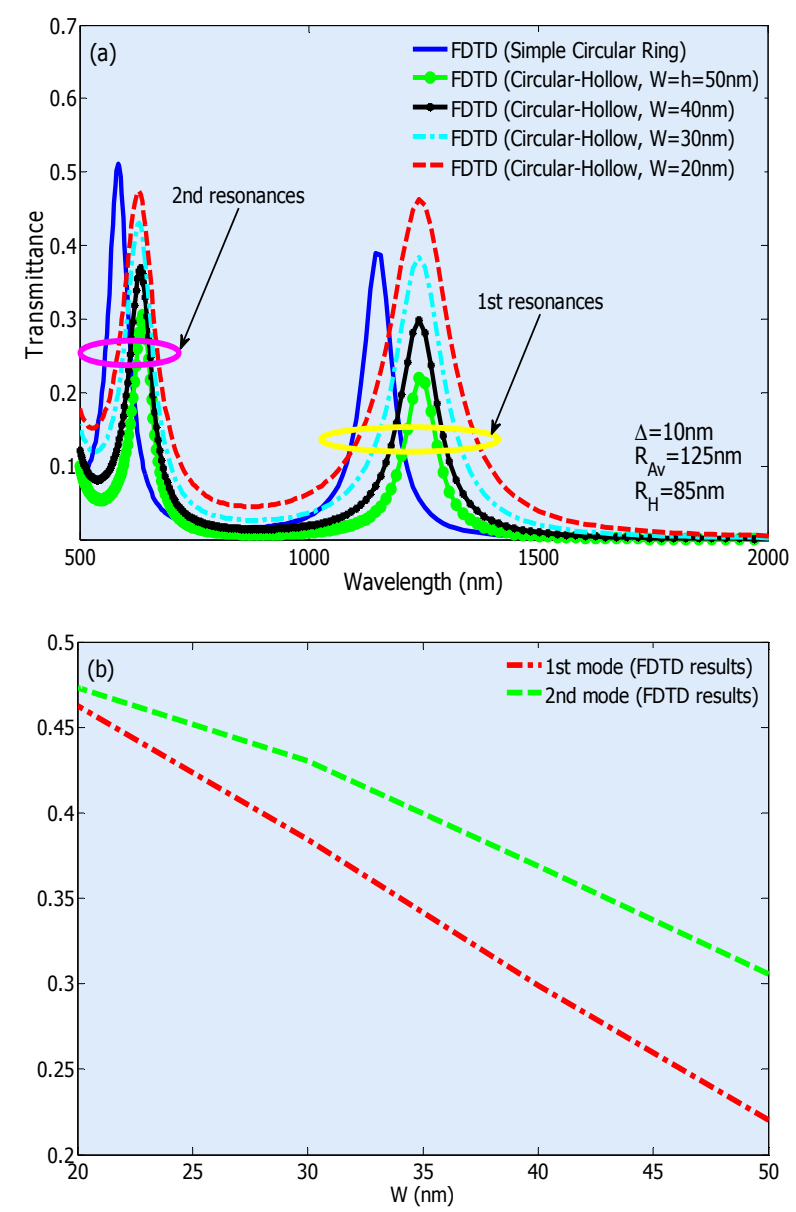

FIG. 8. (a) Transmission spectra of the structure shown in figure 7, for $\left(h=50 \mathrm{~nm}, R_{A v}=125 \mathrm{~nm}, R_{H}=85 \mathrm{~nm}, \Delta=10 \mathrm{~nm}\right)$ and different values of W. (b) Relationship between transmittance of resonance wavelengths and $\mathrm{W}$.

seen from this figure by decreasing $\mathrm{W}$, the coupling area between input/output MIM and ring MIM waveguides increases and therefore more power will be transmitted.

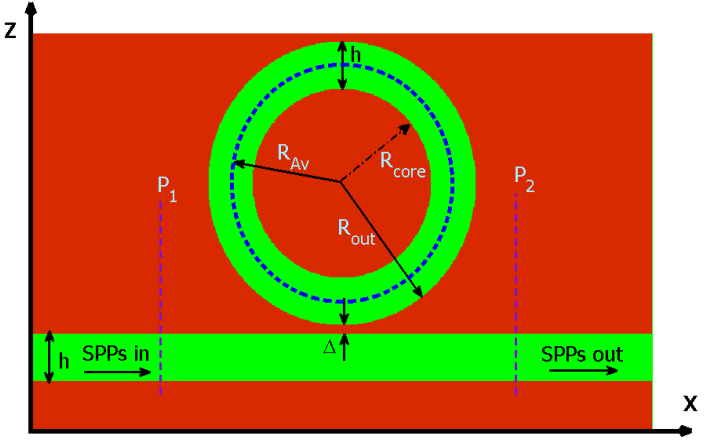

FIG. 9. Schematic of a simple band-stop plasmonic filter consisting of an MIM waveguide coupled to a circular ring resonator. $h$ is set $50 \mathrm{~nm}$.

\section{BAND-STOP PLASMONIC FILTER WITH CIRCULAR RING RESONATOR}

\subsection{Simple Band-stop Waveguide Filter with Circular Ring Resonator}

Another essential and useful component for photonic circuits is a band-stop filter. Figure 9 depicts the geometry of a simple band-stop plasmonic filter which consists of an MIM waveguide laterally coupled to a circular ring resonator. The filter parameters $h, \Delta, R_{\text {core }}, R_{A v}$, and $R_{\text {out }}$ are the same as for the band-pass filter.

Two power monitors are set at points $\mathrm{P}_{1}$ and $\mathrm{P}_{2}$ to observe the incident power $A_{1}$ and the transferred power $A_{2}$. The transmittance of the filter with $R_{A v}=125 \mathrm{~nm}$ and $\Delta=10 \mathrm{~nm}$ is illustrated in figure 10(a). From figure 10(a), it is clear that there are two resonance valleys at $\lambda \approx 1145 \mathrm{~nm}$ and $\lambda \approx 583.5 \mathrm{~nm}$. Figures 10 (b), 10(c) show the field profile of $\left|\mathrm{H}_{\mathrm{y}}\right|$ for the first and second valleys, respectively. As the transmission curve shows, the second resonance mode has a stronger coupling to the ring in comparison with the first one; this is because the wavelength of the second mode is shorter than that of the first one, so that the power density $\left(\mathrm{W} / \mathrm{m}^{2}\right)$ of the second resonance is higher than the first mode. Therefore, more power can be coupled into the ring at the coupling region for the second mode.

\subsection{Band-stop Plasmonic Filter with Hollow-core Ring Resonator}

In this part, by keeping constant the outer dimensions of the band-stop filter (i.e. $\mathrm{R}_{\mathrm{Av}}, \mathrm{h}$ and $\Delta$ ) and hollowing out the core of the ring resonator (see Fig. 11); we check how the resonant wavelengths of the filter will be affected.

As for the band-pass filter case, we anticipate that by increasing $\mathrm{R}_{\mathrm{H}}$, the resonant wavelengths of the band-stop filter experience a red-shift. This case is illustrated in figure 12(a). One can see that by varying $R_{H}$ from $70 \mathrm{~nm}$ to $85 \mathrm{~nm}$, the entire transmission spectrum has undergone a red-shift. Figure 12(b) shows that the wavelength-shifts of the modes 1 and 2 have approximately lineal relations with respect to 

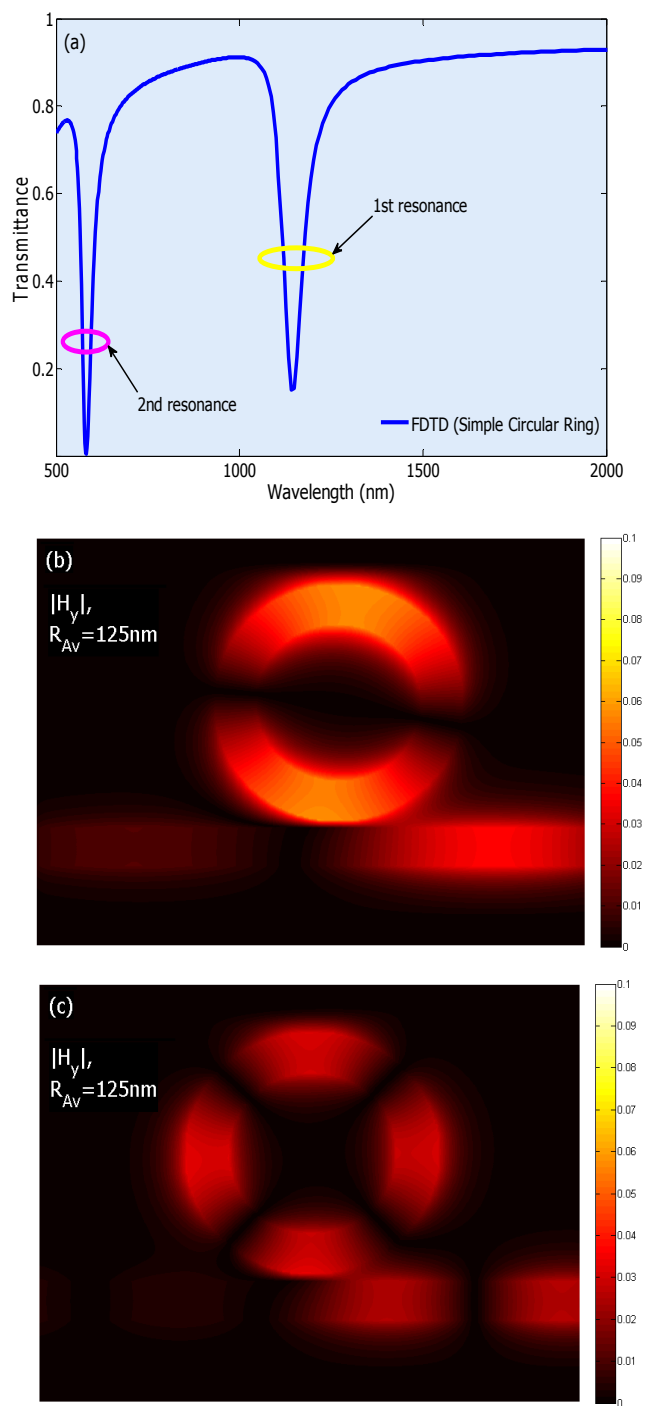

FIG. 10. (a) Transmittance spectrum of the simple band-stop plasmonic filter with circular ring resonator $\left(R_{A v}=125 \mathrm{~nm}, \Delta=\right.$ $10 \mathrm{~nm}$ ). (b) The $\left|\mathrm{H}_{\mathrm{y}}\right|$ field pattern of simple band-stop filter at the first resonance wavelength of $\lambda \approx 1145 \mathrm{~nm}$. (c) The $\left|\mathrm{H}_{\mathrm{y}}\right|$ field profile of the filter at the second resonance wavelength of $\lambda \approx 583.5 \mathrm{~nm}$.

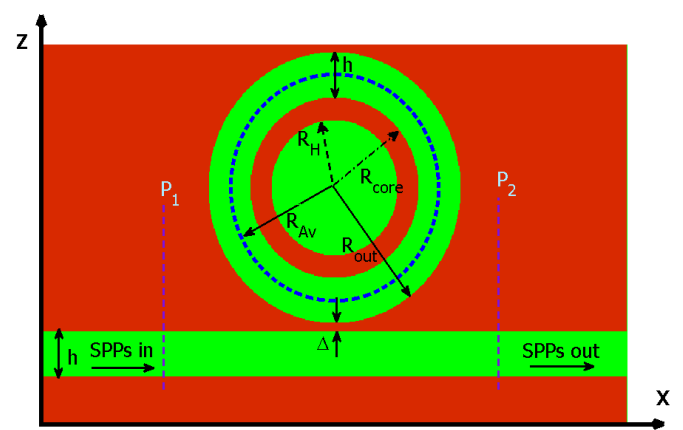

FIG. 11. Schematic of a band-stop plasmonic filter with circular hollow-core ring resonator. ( $h=50 \mathrm{~nm}, R_{A v}=125 \mathrm{~nm}, \Delta$ $=10 \mathrm{~nm}) . R_{H}$ is variable.
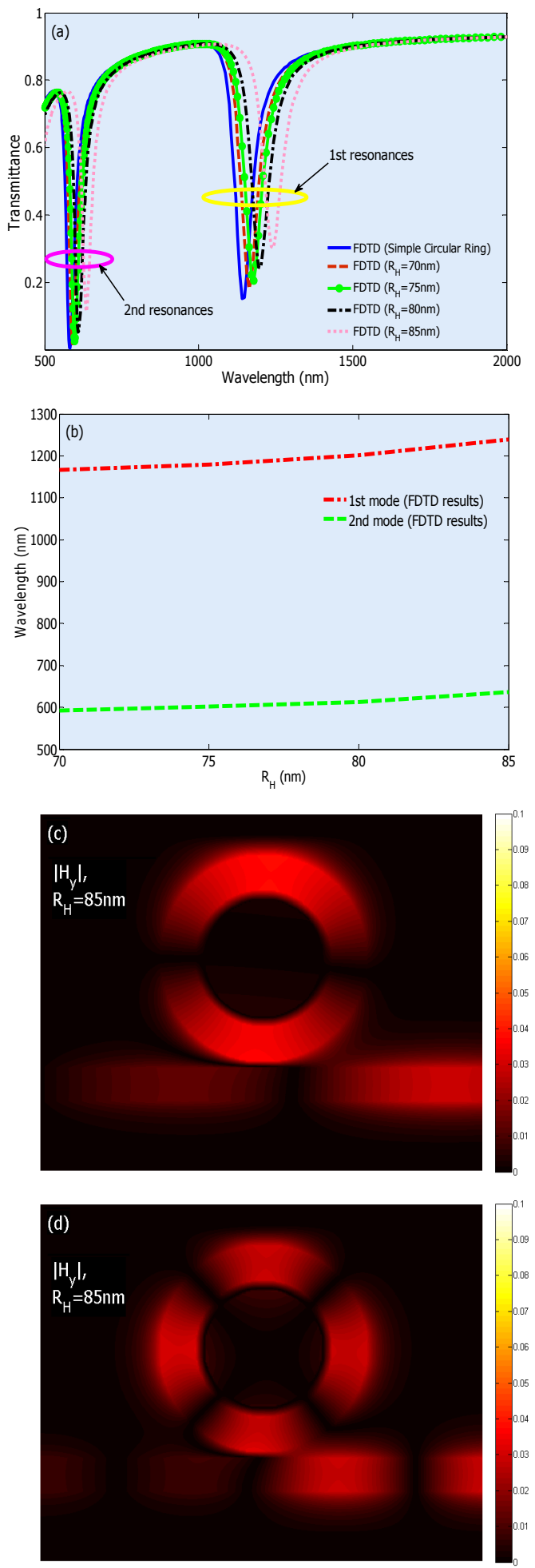

FIG. 12. (a) Transmission spectrum of the band-stop plasmonic filter with circular hollow-core ring resonator shown in figure 11 for different values of $\mathrm{R}_{\mathrm{H}}$. (b) Relationship between resonance wavelengths and hollow radius. (c) The $\left|\mathrm{H}_{\mathrm{y}}\right|$ field pattern of band-stop filter with circular hollow-core ring resonator at the first resonance wavelength of $\lambda \approx 1239$ $\mathrm{nm}$, for $R_{H}=85 \mathrm{~nm}$. (d) The $\left|\mathrm{H}_{\mathrm{y}}\right|$ field pattern of the filter at the second resonance wavelength of $\lambda \approx 636 \mathrm{~nm}$, for $R_{H}=85 \mathrm{~nm}$. 
the variation of hollow radius. This effect is in complete agreement with the case for band-pass filters, i.e. by increasing the resonance volume, the resonant wavelength is increased. So, from the transmittance curves, it is explicit that by altering the $\mathrm{R}_{\mathrm{H}}$, the band-stop filter can be simply adjusted.

\section{CONCLUSION}

In this paper, firstly the propagation characteristics of a band-pass filter composed of two MIM waveguides coupled to each other by a circular ring resonator were studied. To make the proposed filter tunable, an easy way of emptying the core of the ring was introduced and investigated numerically. Then, a band-stop filter consisting of an MIM waveguide laterally coupled to a circular ring resonator was studied and the impact of hollowing out the core of this ring resonator was investigated. It was seen that for both cases, by increasing the radius of the hollow, the resonant wavelengths of the structures experienced a red-shift. A significant advantage of the proposed method of making filters tunable is ease of fabrication. The resonant modes of the structures were calculated by FDTD and it was found that we can easily manipulate the central wavelengths of the resonance transmission by tuning the radius of the hollow while the outer dimensions of the ring are kept constant. The introduced structures can decrease plasmonic filter dimensions and so are potentially a choice for designing of all-optical integrated circuits for optical communication and optoelectronic circuits.

\section{ACKNOWLEDGMENT}

The authors acknowledge the financial support from the Education \& Research Institute for ICT, Iran (Grant No. $500 / 3653)$.

\section{REFERENCES}

1. S. I. Bozhevolnyi, "Plasmonic nanoguides and circuits," in Plasmonics and Metamaterials (Pan Stanford Publishing, Singapore, 2008).

2. E. Ozbay, "Plasmonics: merging photonics and electronics at nanoscale dimensions," Science 311, 189-193 (2006).

3. J. Jung, "Optimal design of dielectric-loaded surface plasmon polariton waveguide with genetic algorithm," J. Opt. Soc. Korea 14, 277-281 (2010).

4. K. M. Byun, "Development of nanostructured plasmonic substrates for enhanced optical biosensing," J. Opt. Soc. Korea 14, 65-76 (2010).

5. S. Kim, Y. T. Byun, D.-G. Kim, N. Dagli, and Y. Chung, "Widely tunable coupled-ring reflector laser diode consisting of square ring resonators," J. Opt. Soc. Korea 14, 38-41 (2010).

6. J. Yoon, G. Lee, S. H. Song, C.-H. Oh, and P.-S. Kim, "Photonic band gaps for surface plasmon modes in dielectric gratings on a flat metal surface," J. Opt. Soc. Korea 6,
76-82 (2002).

7. Z. Fu, Q. Gan, K. Gao, Z. Pan, and F. J. Bartoli, "Numerical investigation of a bidirectional wave coupler based on plasmonic Bragg gratings in the near infrared domain," J. Lightwave Technol. 26, 3699-3703 (2008).

8. D. K. Gramotev and D. F. P. Pile, "Single-mode sub-wavelength waveguide with channel plasmon-polaritons in triangular," Appl. Phys. Lett. 85, 6323-6325 (2004).

9. E. Verhagen, J. A. Dionne, L. Kuipers, H. A. Atwater, and A. Polman, "Near-field visualization of strongly confined surface plasmon polaritons in metal-insulator-metal waveguides," Nano Lett. 8, 2925-2929 (2008).

10. J. Takahara, S. Yamagishi, H. Taki, A. Morimoto, and T. Kobayashi, "Guiding of a one-dimensional optical beam with nanometer diameter," Opt. Lett. 22, 475-477 (1997).

11. K. Leosson, T. Nikolajsen, A. Boltasseva, and S. I. Bozhevolnyi, "Long-range surface plasmon polariton nanowire waveguides for device applications," Opt. Express 14, 314-319 (2006).

12. S. A. Maier, P. G. Kik, H. A. Atwater, S. Meltzer, E. Harel, B. E. Koel, and A. G. Requicha, "Local detection of electromagnetic energy transport below the diffraction limit in metal nanoparticle plasmon waveguides," Nature 2, 229232 (2003).

13. M. Quinten, A. Leitner, J. R. Krenn, and F. R. Aussenegg, "Electromagnetic energy transport via linear chains of silver nanoparticles," Opt. Lett. 23, 1331-1333 (1998).

14. S. A. Maier, P. G. Kik, and H. A. Atwater, "Optical pulse propagation in metal nanoparticle chain waveguides," Phys. Rev. B 67, 205402-1 205402-5 (2003).

15. D. F. P. Pile and D. K. Gramotev, "Channel plasmon-polariton in a triangular groove on a metal surface," Opt. Lett. 29, 1069-1071 (2004).

16. S. I. Bozhevolnyi, V. S. Volkov, E. Devaux, and T. W. Ebbesen, "Channel plasmon-polariton guiding by subwavelength metal grooves," Phys. Rev. Lett. 95, 046802-1 046802-4 (2005).

17. Y. Matsuzaki, T. Okamoto, M. Haraguchi, M. Fukui, and M. Nakagaki, "Characteristics of gap plasmon waveguidewith stub structures," Opt. Express 16, 16314-16325 (2008).

18. S. S. Xiao, L. Liu, and M. Qiu, "Resonator channel drop filters in a plasmon-polaritons metal," Opt. Express 14, 2932-2937 (2006).

19. Q. Zhang, X. G. Huang, X. S. Lin, J. Tao, and X. P. Jin, "A subwavelength coupler-type MIM optical filter," Opt. Express 17, 7549-7554 (2009).

20. A. Hosseini and Y. Massoud, "Nanoscale surface plasmon based resonator using rectangular geometry," Appl. Phys. Lett. 90, 181102 (2007).

21. T. B. Wang, X. W. Wen, C. P. Yin, and H. Z. Wang, "The transmission characteristics of surface plasmon polaritons in ring resonator," Opt. Express 17, 24096-24101 (2009).

22. B. Yun, G. Hu, and Y. Cui, "Theoretical analysis of a nanoscale plasmonic filter based on a rectangular metalinsulator-metal waveguide," J. Phys. D: Appl. Phys. 43, 385102 (2010).

23. H. Lu, X. Liu, D. Mao, L. Wang, and Y. Gong, "Tunable bandpass plasmonic waveguide filters with nanodisk resonators," Opt. Express 18, 17922-17927 (2010).

24. S. A. Maier, Plasmonics: Fundamentals and Applications (Springer, New York, USA, 2007), Chapter 2. 
25. J. A. Dionne, L. A. Sweatlock, H. A. Atwater, and A. Polman, "Plasmon slot waveguides: towards chip-scale propagation with subwavelength-scale localization," Physical Review B 73, 035407-1 035407-9 (2006).

26. K. Y. Kim, Y. K. Cho, H.-S. Tae, and J.-H. Lee, "Light transmission along dispersive plasmonic gap and its subwavelength guidance characteristics," Opt. Express 14, 320-330 (2006).

27. A. D. Rakić, A. B. DjuriŠić, J. M. Elazar, and M. L. Majewski, "Optical properties of metallic films for vertical- cavity optoelectronic devices," Appl. Opt. 37, 5271-5283 (1968).

28. Asanka Pannipitiya, Ivan D.Rukhlenko, Malin premaratne, Haroldo T.Hattori and Govind P. Agrawal, "Improved transmission model for metal-dielectric-metal plasmmonic waveguides with stub structures," Opt. Express 18, 6191-6204 (2010).

29. I. Wolff and N. Knoppik, "Microstrip ring resonator and dispersion measurement on microstrip lines," Electron. Lett. 7, 779-781 (1971). 